\title{
Telemedicine Evaluations in Neuro-Ophthalmology During the COVID- 19 Pandemic: Patient and Physician Surveys
}

Jenna Conway, MD, ${ }^{\mathrm{a}}$ Penina Krieger, MPhil, ${ }^{\mathrm{a}}$ Lisena Hasanaj, BA, ${ }^{\mathrm{a}}$ Linus Sun, MD PhD, ${ }^{\mathrm{b}}$ Jackson M. Scharf, BS, ${ }^{\mathrm{b}}$ Jeffrey G. Odel, MD, ${ }^{\mathrm{b}}$ Marc J. Dinkin, MD, ${ }^{\mathrm{c}}$ Cristiano Oliveira, MD, ${ }^{\mathrm{c}}$ Devin D. Mackay, MD, ${ }^{\mathrm{d}}$ Nailyn Rasool, MD, ${ }^{\mathrm{g}}$ Melissa Ko, MD, ${ }^{\mathrm{d}}$ Janet C. Rucker, MD, ${ }^{\mathrm{a} e \mathrm{e}}$ Steven L. Galetta, MD, ${ }^{\mathrm{a}, \mathrm{e}}$ Laura J. Balcer, MD MSCE, ${ }^{\mathrm{a}, \mathrm{e}, \mathrm{f} *}$

*Departments of ${ }^{\text {a }}$ Neurology, NYU Grossman School of Medicine, New York, NY, USA;

${ }^{\mathrm{b}}$ Neurology and Ophthalmology, Columbia University Medical Center, New York, NY, USA;

${ }^{\mathrm{c} N e u r o l o g y}$ and Ophthalmology, Cornell University Medical Center, New York, NY, USA;

${ }^{\mathrm{d} N e u r o l o g y, ~ O p h t h a l m o l o g y}$, and Neurological Surgery, Indiana University School of Medicine, Indianapolis, IN, USA; ${ }^{g}$ Department of Ophthalmology, University of California, San Francisco, San Francisco, CA, USA; ' $O$ Ophthalmology, NYU Grossman School of Medicine, New York, NY, USA; ${ }^{\mathrm{f} P o p u l a t i o n}$ Health, NYU Grossman School of Medicine, New York, NY, USA

* Corresponding author: $\quad$ Laura J. Balcer, MD, MSCE

Department of Neurology

NYU Grossman School of Medicine

222 E. 41st Street, 14th Floor,

New York, NY 10017

Tel. +6465017681

Fax: +2122637721

Email:laura.balcer@nyulangone.org

Key Words: Telemedicine

Virtual health visits

Neuro-ophthalmology

Patient satisfaction

Provider satisfaction

Conflict of interest: The authors have no conflicts of interest.

Funding: This study was supported in part by the NYU Grossman School of Medicine.

Keyword count: 5

Abstract word count: 368 words

Manuscript word count: 1,506 words

Tables: 2

Figures: 0

This is the author's manuscript of the article published in final edited form as:

Conway, J., Krieger, P., Hasanaj, L., Sun, L., Scharf, J. M., Odel, J. G., Dinkin, M. J., Oliveira, C., Mackay, D. D., Rasool, N., Ko, M., Rucker, J. C., Galetta, L., \& Balcer, L. J. (2021). Telemedicine Evaluations in Neuro-Ophthalmology During the COVID- 19 Pandemic:

Patient and Physician Surveys. Journal of Neuro-Ophthalmology, 18. 
Tele-Neuro-Ophthalmology During COVID-19

\section{Abstract}

Background: The novel coronavirus 2019 (COVID-19) pandemic has transformed healthcare.

With the need to limit COVID-19 exposures, telemedicine has become an increasingly important format for clinical care. Compared to other fields, neuro-ophthalmology faces unique challenges given its dependence on physical examination signs that are difficult to elicit outside the office setting. As such, it is imperative to understand both patient and provider experiences in order to continue to adapt the technology and tailor its application. The purpose of this study is to analyze both neuro-ophthalmology physician and patient satisfaction with virtual health visits during the time of the COVID-19 pandemic.

Methods: Across three institutions (NYU Langone Health, Indiana University Health, and Columbia University Medical Center), telemedicine surveys were administered to 159 patients. Neuro-ophthalmologists completed 157 surveys; each of these were linked to a single patient visit. Patient surveys consisted of five questions regarding visit preparation, satisfaction, challenges, and comfort. The physician survey included four questions that focused on ability to gather specific clinical information by history and examination.

Results: Among 159 patients, 104 (65.4\%) reported that they were satisfied with the visit, and 149 (93.7\%) indicated that they were comfortable asking questions. Sixty-eight (73.9\%) patients found the instructions provided prior to the visit easy to understand. Potential areas for improvement noted by patients included more detailed preparation instructions and better technology (phone positioning, internet connection, software). Over 87\% (137/157) of neuroophthalmologists surveyed reported having performed an examination that provided enough information for medical decision-making. Some areas of the neuro-ophthalmologic exam were reported to be easy to conduct (range of eye movements, visual acuity, Amsler grids, Ishihara 
color plates, and pupillary exam). Other components were more difficult (saccades, red desaturation, visual fields, convergence, oscillations, ocular alignment, and smooth pursuit); some were especially challenging (vestibulo-ocular reflex [VOR], VOR suppression, and optokinetic nystagmus). Clinicians noted that virtual health visits were limited by patient preparation, inability to perform certain parts of the examination (funduscopy and pupils), and technological issues.

Conclusions: Among virtual neuro-ophthalmology visits evaluated, most offer patients with appointments that satisfy their needs. The majority of physicians in this cohort obtained adequate clinical information for decision-making. Even better technology and instructions may help improve aspects of virtual health visits. 
Tele-Neuro-Ophthalmology During COVID-19

\section{Introduction}

Telemedicine, the delivery of remote healthcare services where patients and providers are physically separated, has historically been underutilized in healthcare. One of the earliest uses of hospital-based telemedicine was in the late 1950s, when a closed-circuit television link was used by the Nebraska Psychiatric Institute and Norfolk State Hospital for psychiatric consultations (1). Ultimately, the advent of high-speed internet led to advances in telemedicine. Remote, or nonface-to-face, visits became more feasible with increased access to portable devices, such as laptop computers and mobile phones (2). Despite advances in technology and the proven utility of telemedicine, the widespread use of telemedicine has remained relatively low (3). This is particularly true for the field of neuro-ophthalmology, which may be explained by various factors such as infrastructure support, reimbursement, and the unique challenges that neuroophthalmology faces given its dependence on both ocular and neurological examination signs that are difficult to elicit outside the office setting (4).

The novel coronavirus 2019 (COVID-19) pandemic has transformed healthcare. With the need to limit COVID-19 exposures, telemedicine has become an increasingly prevalent and convenient format for clinical care. Healthcare providers and medical institutions have responded to concerns of exposure to COVID-19 by offering remote appointments as a means to provide care (5). For instance, NYU Langone Health, at the epicenter of the COVID-19 pandemic in New York City, had more than 5,000 virtual health visits conducted daily within one month of the pandemic onset in March 2020 (4).

With the larger scale use of this relatively new technology during the COVID-19 pandemic, it is imperative to understand the patient and provider experiences and to continue to adapt the technology and tailor its application. The purpose of this study was to analyze both 
Tele-Neuro-Ophthalmology During COVID-19

neuro-ophthalmology physician and patient satisfaction with virtual health visits during the initial period of the COVID-19 pandemic in the United States.

\section{Methods}

Study Participants

Three institutions, including New York University (NYU) Langone Health, Indiana University Health, and Columbia University Medical Center, provided surveys to neuroophthalmology physicians and patients from April 2020 to July 2020. A total of 12 neuroophthalmology physicians participated in the study comprised of 8 from NYU Langone Health, 2 from Indiana University Health, and 2 from Columbia University Medical Center. A total of 159 patient surveys and 157 neuro-ophthalmology physician surveys were completed (57 from NYU Langone Health, 35 from Indiana University Health, and 67 from Columbia University Medical Center). Each survey had a patient and physician component. At the time of the study, participating institutions offered all visits as virtual as there were limitations in the number of inperson visits allowed, which were reserved for urgent cases. In addition, only video visits were included in this study. The study protocol was approved by the NYU Grossman School of Medicine, Indiana University School of Medicine, and Columbia University Medical Center Institutional Review Boards (IRBs). All participants provided verbal consent using an IRBapproved script that was read to them following completion of their virtual health visits.

Demographics for participating neuro-ophthalmology physicians were captured. 
Tele-Neuro-Ophthalmology During COVID-19

The EyeHandbook application, which is free for smartphone and tablet users, was used to perform some components of the exam. Specifically, visual acuity, Amsler grids, and Ishihara color plates were assessed using the application. The EyeHandbook application has been validated against the Ishihara color vision test in two clinical studies $(6,7)$.

Survey

Following the virtual patient-physician encounter, the patient and physician completed linked surveys, which were developed by the physicians who participated in this study. The physician who performed the virtual visit administered the survey to the patient verbally at the end of the encounter. Physician surveys could not be completed at one study site. The patient survey consisted of five questions (Table 1). Patients were asked how easy it was to understand the instructions to prepare for their virtual health visit, if they felt that the virtual health visit satisfied their needs (versus if they felt that they still needed to be seen in-person), if there was anything that would have helped them to better prepare for their virtual health visit, if there were any particular aspects of the virtual health visit that they found challenging, and how comfortable they were with the virtual health visit (compared to an in-person visit) with regard to asking questions about their health. Free text questions on the patient survey included what would have helped them to better prepare for the visit and what aspects of the virtual health visit they found challenging.

The physician survey included four questions related to exam findings that could conceivably be conducted remotely (Table 1). Neuro-ophthalmologists were asked if they were able to complete an examination as part of the virtual health visit that provided enough information for medical decision-making. They were also asked what parts of the examination 
they found surprisingly easy to gather useful information from, which comprised visual acuity, Amsler grids, color plates, red desaturation, pupils (size, shape, and afferent pupillary defect), visual fields, range of eye movements, ocular alignment, saccade speed and accuracy, smooth pursuit, vestibulo-ocular reflex [VOR], VOR suppression, convergence, opto-kinetic nystagmus [OKN], and ocular oscillations such as nystagmus and saccadic intrusions. In addition they were asked if there were any aspects that could help enhance the quality of the information obtained virtually and approximately how many virtual health visits they had performed to date using the current platforms. On the physician survey, free text questions included what aspects of the physical exam were inadequate and/or needed to be performed in-person, and any aspects that could help enhance the quality of information obtained virtually.

Analysis

Responses to categorical questions in the survey were reported as proportions. Responses to free text survey questions were reported qualitatively and were analyzed qualitatively through thematic grouping.

Results

Participating neuro-ophthalmology physicians were 50\% male and 50\% female with a median age of 46 years (range 36-71). Survey results are reported in Table 2. Among 159 patients, 104 (65.4\%) reported that the visit had satisfied their needs. Most (149, 93.7\%) reported being as comfortable asking questions related to their health as they would be in a faceto-face appointment. Sixty-eight patients (73.9\%) also found that instructions provided prior to the virtual health visit were very easy to understand. Some areas of improvement were 
highlighted by the responses, including continued improvement of instructions and preparation for the virtual health visit as well as improvements in technology. Respondents noted phone positioning, connectivity, and software posed challenges and expressed concerns regarding the breadth and detail of the exam given the remote nature.

Eighty-seven percent (137/157) of surveys completed by neuro-ophthalmologists reported that their examinations provided enough information for medical decision-making. Some areas of the neuro-ophthalmologic examination were reported to be "surprisingly easy" to conduct with regard to virtually gathering useful information in encounters; these included range of eye movements (80.3\%), visual acuity (74.5\%), Amsler grids (58.0\%), Ishihara color plates (50.3\%), and pupil exam (45.9\%). Other components of the examination proved to be more difficult, including saccades (36.9\%), red desaturation (35.7\%), visual fields (29.9\%), convergence (22.3\%), assessment of ocular oscillations (21.7\%), ocular alignment (21.7\%), and smooth pursuit (21.7\%). VOR (1.9\%), VOR suppression (0.6\%), and OKN (0.0\%) were especially challenging to examine virtually. Physicians noted that virtual health visits were limited by patients’ preparation prior to visits, inability to perform funduscopic examinations, challenges with the pupillary exam, and issues related to phone positioning and internet connections.

\section{Discussion}

Results of this study demonstrate that virtual health visits are generally well-received by both patients and clinicians in neuro-ophthalmology. The surveys in our study were particularly helpful in identifying aspects of the neuro-ophthalmologic examination that could be improved with more technologically-accessible methods. 
The COVID-19 pandemic resulted in widespread adoption of telemedicine in various specialties including neuro-ophthalmology practice $(8,9)$. With rising patient and provider interest in telemedicine, social distancing practices, changing regulatory restrictions, and increased reimbursement, many neuro-ophthalmologists have offered virtual health visits (10). Despite this sudden change in the mode of healthcare delivery, our study demonstrates that neuro-ophthalmology patients were mostly satisfied with virtual health visits. A majority of patients (65\%) reported that their visit had satisfied their needs, despite it not being an in-person appointment. This proportion appears to be less than that reported by some other specialties during the COVID-19 pandemic $(11,12)$, and may be due to a necessarily more extensive physical examination in neuro-ophthalmology compared to other specialties. In our study, patients reported concerns with the breadth and detail of the exam given the remote nature. Despite the platform for virtual health visits being the patients' and physicians' smart phones or tablets, some areas for improvement in neuro-ophthalmology virtual health visits that patients noted included more approachable technologies and instructions to prepare for the visit.

Notwithstanding the heavy reliance on the physical examination by neuroophthalmologists (4), 87\% of the time, neuro-ophthalmologists reported having performed an examination during the COVID-19 pandemic that provided enough information for medical decision-making. This may be due to the fact that much of the neuro-ophthalmologic exam can be performed externally to the eye itself, in contrast to other subspecialties of ophthalmology (13). In our study, areas of the neuro-ophthalmologic examination that were surprisingly easy to conduct virtually according to physicians during the COVID-19 pandemic included eye movements, visual acuity, Amsler grids, Ishihara color plates, and the pupil exam. In contrast, other components of the examination proved to be more difficult (saccades, assessment of red 
desaturation, visual fields, convergence, oscillations, ocular alignment, and smooth pursuit). Some were especially challenging (VOR, VOR suppression, and OKN). Physicians noted that virtual health visits were limited by the patient's preparation, inability to perform certain aspects of the examination (funduscopy and pupils), and technological issues with connections to patients. These results are similar to those of a recent study of telehealth adoption by neuroophthalmologists during COVID-19. This study found that telemedicine was most helpful for conditions that relied most on history, external examination, and previously collected ancillary testing, while conditions requiring a funduscopic exam were not as helpful (9).

There are numerous limitations of this study. One important limitation is the generalizability of this study. While there were not any specific selection criteria as all patients were offered virtual visits, it is possible that specific providers and patients were more likely to use telemedicine at the time this study was conducted during the COVID-19 pandemic and thus selection bias may be present. An additional limitation is that some parts of the exam (e.g., OKN) were not performed on every patient. Furthermore, the provider who completed each survey was not recorded; it may be useful to analyze the proportions for each provider since ease of performing exam components virtually may vary among providers. In the future, it would also be interesting to analyze provider age with the perception that enough information was gathered. In addition, it may be useful to analyze patient demographics in terms of satisfaction with the virtual visit, however, these were not routinely collected. Finally, patient surveys were performed verbally by the examining neuro-ophthalmologist, which may bias patients to give more favorable responses. Future studies that assure anonymity for the patient would be useful.

\section{Conclusions}


The COVID-19 pandemic has driven rapid expansion of telemedicine use in neuroophthalmology beyond historical levels. This reflects important changes in the delivery of healthcare. Virtual neuro-ophthalmology visits offer most patients with appointments that satisfy their needs and the majority of providers obtain adequate clinical information for decisionmaking. Better technology and instructions may help improve aspects of virtual visits, and some conditions still require in-person appointments for proper management. 


\section{References}

1. Field MJ. Telemedicine: A guide to assessing telecommunications for health care: National Academies Press; 1996.

2. Maheu M, Whitten P, Allen A. E-Health, Telehealth, and Telemedicine: a guide to startup and success: John Wiley \& Sons; 2002.

3. Kane CK, Gillis K. The use of telemedicine by physicians: still the exception rather than the rule. Health Affairs. 2018;37(12):1923-30.

4. Grossman SN, Calix R, Tow S, Odel JG, Sun LD, Balcer LJ, et al. Neuro-Ophthalmology in the Era of COVID-19: Future Implications of a Public Health Crisis. Ophthalmology. 2020.

5. Klein BC, Busis NA. COVID-19 is catalyzing the adoption of teleneurology. AAN Enterprises; 2020.

6. Ozgur OK, Emborgo TS, Vieyra MB, Huselid RF, Banik R. Validity and acceptance of color vision testing on smartphones. Journal of Neuro-ophthalmology. 2018;38(1):13-6.

7. Shah AA, Pasadhika S, Kim J, Wang M. Pseudoisochromatic color vision testing on an iPhone. Investigative Ophthalmology \& Visual Science. 2012;53(14):6399-.

8. Mann DM, Chen J, Chunara R, Testa PA, Nov O. COVID-19 transforms health care through telemedicine: evidence from the field. Journal of the American Medical Informatics Association. 2020.

9. Moss HE, Lai KE, Ko MW. Survey of telehealth adoption by neuro-ophthalmologists during the COVID-19 pandemic: Benefits, barriers, and utility. Journal of NeuroOphthalmology. 2020.

10. Ko MW, Busis NA. Tele-Neuro-Ophthalmology: Vision for 20/20 and Beyond. Journal of Neuro-Ophthalmology. 2020.

11. Layfield E, Triantafillou V, Prasad A, Deng J, Shanti RM, Newman JG, et al. Telemedicine for head and neck ambulatory visits during COVID-19: Evaluating usability and patient satisfaction. Head \& Neck. 2020.

12. Mustafa SS, Yang L, Mortezavi M, Vadamalai K, Ramsey A. Patient satisfaction with telemedicine encounters in an allergy/immunology practice during the COVID-19 pandemic. Annals of Allergy, Asthma \& Immunology. 2020.

13. Saleem SM, Pasquale LR, Sidoti PA, Tsai JC. Virtual ophthalmology: telemedicine in a Covid-19 era. American Journal of Ophthalmology. 2020. 
Table 1. Survey

\section{TELEMEDICINE EXPERIENCE FOR NEURO-OPHTHALMOLOGY IN THE ERA} OF THE COVID-19 PANDEMIC

Thank you very much for completing these surveys as part of our research study! The first survey should be completed at the end of the virtual health visit with the patient still online; the second should be completed by the provider following the virtual health visit.

QUESTIONS TO ASK THE PATIENT:

1. How easy was it to understand the instructions to prepare for your virtual health visit?
a. Very easy
b. Somewhat easy
c. Somewhat difficult
d. Very difficult

2. Do you feel that the virtual health visit satisfied your needs, or did it feel like you still needed to be seen in-person?

a. Virtual health visit satisfied my needs

b. Felt like I still needed to be seen in-person

3. Is there anything that would have helped you to better prepare for your virtual health visit?

a. No

b. Yes; if yes, what would have helped?

4. Were there any particular aspects of the virtual health visit that you found challenging?
a. No
b. Yes; if yes, what aspects did you find challenging? 
5. How comfortable were you with the virtual health visit compared to an in-person visit with regard to asking questions about your health?
a. Very comfortable
b. Somewhat comfortable
c. Somewhat uncomfortable
d. Very uncomfortable

Thank you very much for completing this survey!

\section{QUESTIONS FOR YOU TO ANSWER AS THE PROVIDER:}

6. Were you able to complete an examination as part of the virtual health visit that provided enough information for medical decision-making?

a. Yes

No; if no, what aspects were inadequate and/or needed to be determined or performed inperson?

7. What parts of the examination did you find surprisingly easy to gather useful information from? Please circle all that apply.
a. Visual acuity
b. Amsler grids
C. Color plates (Ishihara)
d. Red desaturation
e. Pupils 
f. Visual fields

g. Range of eye movements

h. Ocular alignment

i. Saccade speed and accuracy

j. Smooth pursuit

k. VOR

l. VOR suppression

m. Convergence

n. OKN

o. Ocular oscillations (nystagmus, saccadic intrusions)

8. Are there any aspects that could help enhance the quality of the information obtained virtually? (positioning of the phone cameras or lighting, for example)

a. No

b. Yes; if yes, what could have helped?

9. Approximately how many virtual health visits have you performed to date using the current platforms?

Thank you very much for completing the provider survey! 
Tele-Neuro-Ophthalmology During COVID-19

Table 2. Summary data from patient and physician survey responses.

\begin{tabular}{|c|c|}
\hline Questions & Positive Responses (\%) \\
\hline \multicolumn{2}{|l|}{ Patient survey $(\mathrm{n}=159)$} \\
\hline $\begin{array}{l}\text { Ease in understanding instructions to prepare for } \\
\text { visit (n=92) } \\
\text { (very easy; somewhat easy; somewhat difficult; } \\
\text { very difficult) }\end{array}$ & $\begin{array}{l}68(73.9 \%) ; 17 \\
1(1.1 \%)\end{array}$ \\
\hline Virtual health visit satisfied needs & $104(65.4 \%)$ \\
\hline Anything that would help prepare for visit & $39(24.5 \%)$ \\
\hline Particular aspects of visit found challenging & \\
\hline $\begin{array}{l}\text { Comfort asking questions about health } \\
\text { (very comfortable; somewhat comfortable; } \\
\text { somewhat uncomfortable; very uncomfortable) }\end{array}$ & ); 8 (5.0\%); 2 (1.3\%); 0 (0\%) \\
\hline \multicolumn{2}{|l|}{ Physician survey $(\mathrm{n}=157)$} \\
\hline $\begin{array}{l}\text { Enough information gathered to make m } \\
\text { decisions }\end{array}$ & $87.3 \%)$ \\
\hline Easy gather information: visual acuity & $117(74.5 \%)$ \\
\hline Easy gather information: Amsler grids & $91(58.0 \%)$ \\
\hline Easy gather information: Ishihara colors plates & $79(50.3 \%)$ \\
\hline Easy gather information: red desaturation & $56(35.7 \%)$ \\
\hline Easy gather information: pupils & $72(45.9 \%)$ \\
\hline Easy gather information: visual fields & $47(29.9 \%)$ \\
\hline Easy gather information: range of eye movements & $126(80.3 \%)$ \\
\hline Easy gather information: ocular alignment & $34(21.7 \%)$ \\
\hline Easy gather information: saccade speed/accuracy & $58(36.9 \%)$ \\
\hline Easy gather information: smooth pursuit & $34(21.7 \%)$ \\
\hline Easy gather information: VOR & $3(1.9 \%)$ \\
\hline
\end{tabular}


Tele-Neuro-Ophthalmology During COVID-19

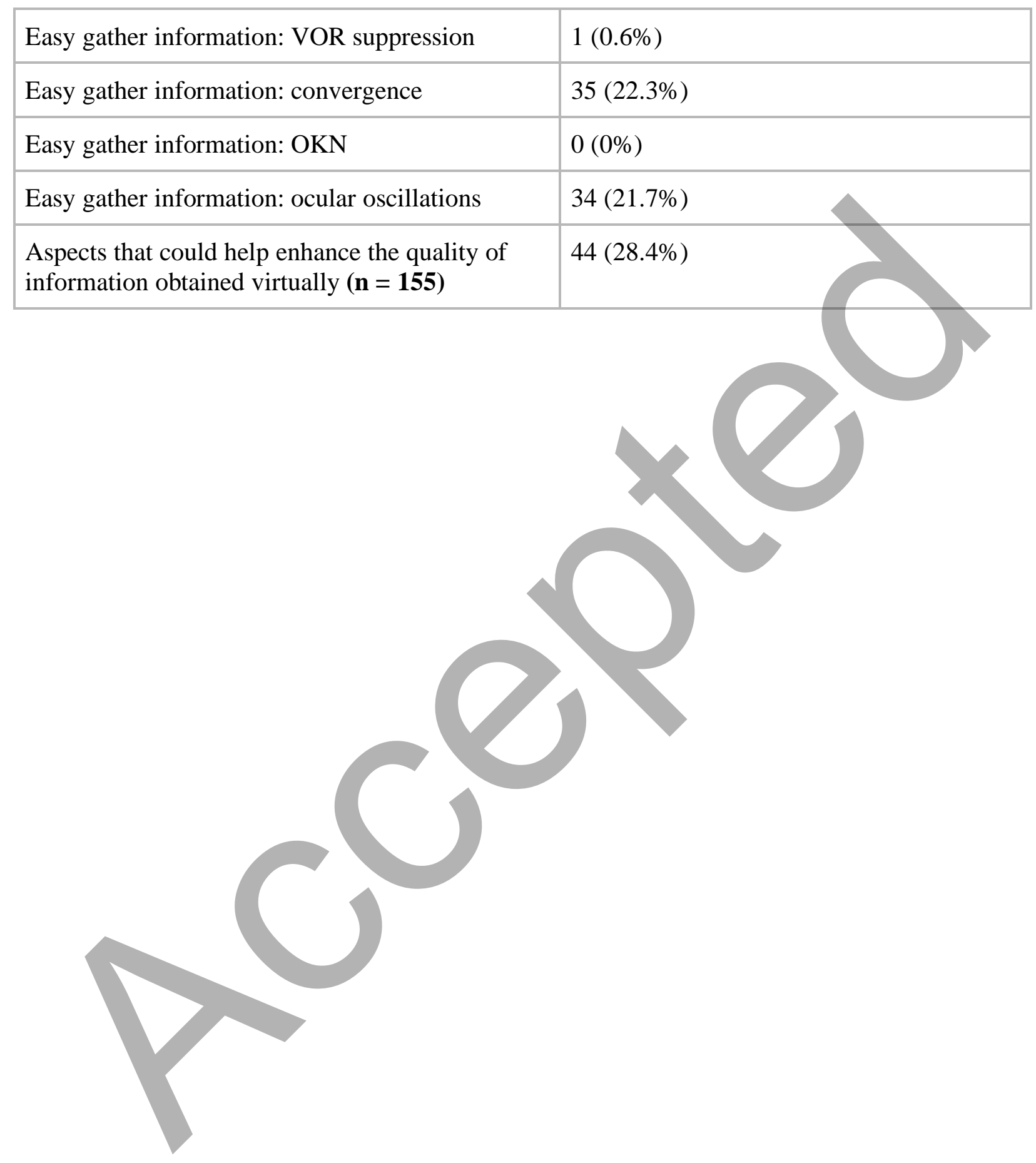


Tele-Neuro-Ophthalmology During COVID-19

\section{Statement of Authorship}

1. Category 1:

a) Conception and design

Jenna Conway

Jeffrey Odel

Marc Dinkin

Linus Sun

Melissa Ko

Janet Rucker

Steven Galetta

Laura Balcer

b) Acquisition of data

All authors

c) Analysis and interpretation of data

Jenna Conway

Penina Krieger

Lisena Hasanaj

Linus Sun

Melissa Ko

Laura Balcer

2. Category 2:

a) Drafting the manuscript

Jenna Conway

Penina Krieger

Steven Galetta

b) Revising it for intellectual content

All authors

3. Category 3:

a) Final approval of the completed manuscript

All authors 